\title{
MERLIN OBSERVATIONS OF SEYFERT NUCLEI
}

\author{
A. PEDLAR \& M.J. KUKULA
}

NRAL, University of Manchester, Jodrell Bank, Nr Macclesfield, Cheshire, UK. SK11 9DL

Radio emission from Seyfert nuclei appears to be intimately related to narrow line region (NLR) of ionised gas. Both regions have an extent of a few hundred parsecs corresponding to typically a few arcsec, and are only marginally resolved by ground based observations. HST and adaptive optics are giving optical images of the ionised gas with angular resolutions of order $0.1^{\prime \prime}$. It is essential that high quality radio images are available with similar resolution so that models relating the two regions can be tested. The extended MERLIN is ideally suited for this task. It has angular resolutions of $0.05^{\prime \prime}$ at $5 \mathrm{GHz}$ and $0.13^{\prime \prime}$ at $1.5 \mathrm{GHz}$ and sensitivities of a few $10 \mathrm{~s}$ of $\mu \mathrm{Jy}$. In this contribution we shall summarise the results on two objects.

The 5Ghz MERLIN image of NGC4151 (Pedlar et al MNRAS 263 471, 1993) confirms the picture of a two sided jet along $\mathrm{Pa} \sim 77^{\circ}$. By comparison with a MERLIN $1.5 \mathrm{GHz}$ image it is clear that most of the source has a steep radio spectral index. However the eastern component of the central double has a relatively flat index, consistent with it being coincident with the optical continuum nucleus. The radio collimation does not appear to align with the elongation of [OIII] emission on similar scales seen with the HST.

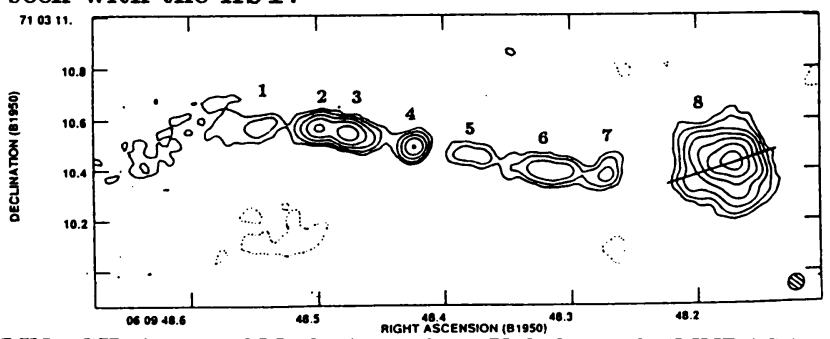

Fig. 1. MERLIN $5 \mathrm{GHz}$ image of Markarian 3 from Kukula et al. (MNRAS in press)

Markarian 3 shows the finest example of a radio jet in a Seyfert nucleus. We identify component 4 (See Fig. 1) as the nucleus both from its relatively flat spectral index spectral index and its compact ( $<30 \mathrm{mas})$ structure. This implies we have a two sided jet extending $\sim 300 \mathrm{pc}$ either side of the nucleus. The inner jets are quite linear, but bend significantly beyond $150 \mathrm{pc}$ resulting in an 'S' type symetry. The radio image is strikingly similar to HST images in [OIII] (Boksenberg and Catchpole -Private communication), although the [OIII] ' $S$ ' is measurably smaller than the radio. We are investigating a model in which a bowshock, of the type discussed by Taylor et al (MNRAS 255 351, 1991), is moving into a rotating, rather than stationary, ambient medium could produce this effect.

T. J.-L. Courvoisier and A. Blecha: Multi-Wavelength Continuum Emission of AGN, 430.

(C) 1994 IAU. Printed in the Netherlands. 